\title{
13
}

\section{Chemical Exposure: An Overview}

\author{
JAMES E. LESSENGER
}

Key words: pesticides, growth regulators, fertilizers, nutrients, buffers, petroleum products

Agricultural chemicals comprise thousands of formulations, including petroleum products, pesticides, growth regulators, buffers, nutrients and fertilizers, and veterinary medications. These chemicals may be used as solids in granular, powder, pellet, or block form; liquids in mists and sprays; or in gaseous forms as fumigants or fuels. Application of chemicals to crops may be by sprays of liquids from aircraft or ground machines; broadcast of solids from aircraft, vehicles, or stationary sources; injection of gas, liquid, or solids into water, soil, animals, or feed; or gaseous exposure in fumigation cells. Animals may be dipped in pools of dilute insecticides to remove surface insects. To save on manpower and fuel, it is common to apply five or more chemicals at once to a crop, making it difficult to determine which are the relevant agents. Mass casualty situations may result from the sudden release of large quantities of chemicals from a manufacturing, storage, or transportation facility, or from the group's perception of a release as in mass psychogenic hysteria (Table 13.1) (1-3).

Exposure does not necessarily equal poisoning. If a person is working in an area where a chemical is being used, he or she may not be exposed. Exposure to a chemical may not mean there will be enough external or internal contact to produce the physiological changes of poisoning. Poisoning may not produce a clinical level of illness, impairment, or disability. It is a mistake to assume a person has become ill from a chemical just because he or she was present in the vicinity where it was thought to have been used. It is important to consider the differential diagnoses of chemical illness when evaluating an alleged chemical injury for causation (1).

The massive amount of information and misinformation in the public media about farm chemicals complicates the evaluation of the patient. Some chemicals produce a particularly noxious odor that can cause nausea or anxiety about exposure, yet cause none of the physiological processes of poisoning. Dursban ${ }^{\mathrm{TM}}$, for example, has a particularly obnoxious odor, and small 
TABLE 13.1. Farm chemicals, pesticides, and other chemical agents $(1,2,16)$.

\begin{tabular}{lc}
\hline Farm chemicals & Rodenticides \\
Gasoline fuels & Coumadin and other anticoagulants \\
Diesel fuels & Strychnine \\
Jet fuels & Sodium fluoroacetate \\
Oils and lubricants & Fungicides \\
Fluids (hydraulic, etc) & Carbamates \\
Kerosene (mixed with chemicals as & Organophosphates \\
applied to crops to make the pesticides & Others, including sulfur, captan, captofol \\
settle on the leaves) & Antimicrobials (disinfectants) \\
Pesticides (Chapter 16) & Triazine-S-triones, \\
Insecticides & Chlorine-releasing agents \\
Organochlorines & Chlorine \\
Organophosphates & Dichloronitrobenzene \\
Carbamates & Growth regulators (Chapter 15) \\
Pyrethrins & Plant regulators \\
Synthetic pyrethroids & Insect regulators \\
Nicotine & Buffers (to bring chemical mixtures to \\
Rotenone & neutral pH before application) \\
Microbiologicals (Bacillus & Nutrients and fertilizers (Chapter 14) \\
thuringiensis) & Elemental compounds \\
Elemental substances (sulfur) & Anhydrous ammonia \\
Herbicides & Gypsum \\
Trichloro/dichlorophenoxyherbicides & Others \\
Urea derivatives & Veterinary medications \\
Carbamates & Immunizations \\
Triazines & Antibiotics \\
Glyphosate & Hormones to promote growth \\
& and production \\
&
\end{tabular}

Source: Data from Lessenger (1), O’Malley (2), Tordoir et al. (4), Reigart and Roberts (5).

amounts can cause anxiety and fear in people when no significant exposure has occurred. As urban growth encroaches upon farmland, more and more people live adjacent to farms and farm animals. Unpleasant odors familiar to those people working in agriculture may be misinterpreted by new arrivals as dangerous toxicants. A careful history and physical examination can differentiate fears and anxiety from actual poisoning $(6,7)$.

\section{Clinical Presentation}

An emergent presentation is seen in a person who is exposed to a chemical and becomes immediately ill and thinks agricultural chemicals are to blame. Most emergent chemical-related illnesses are seen in the hospital emergency department. A nonemergent situation is seen by many physicians in their offices where the patient may be sent after an emergency department visit. Alternatively, the patient may arrive directly from the workplace, either the 
same day of the exposure or sometime later. The vital signs are typically stable and there may be a question whether the person was actually exposed or poisoned. The emergency department may not have the time to do a thorough history or have the resources of past records, private investigators' reports, governmental reports, site visits, and research to aid in the diagnosis and the determination of causation, impairments, disabilities, or future medical care. The office-based physician is often asked to determine these issues $(1,7,8)$.

\section{Case Study 1}

A 34 -year-old man who works as a certified pesticide applicator became profoundly vertiginous and collapsed while applying hydrogen cyanamide, a growth regulator applied to promote uniform budding in citrus. He was wearing a full protective ensemble, including overalls, boots, gloves, mask, goggles, and helmet. The patient was questioned extensively because hydrogen cyanamide can cause an Antabuse reaction when used by a person who has recently consumed alcohol or used alcohol-containing products. He denied using alcohol in any way, including after-shave and hair tonic.

In accordance with longstanding company protocols, coworkers removed the employee from the field, removed the protective ensemble, and decontaminated him with water. An ambulance was called and emergency medical technicians (EMTs) responded. They donned full protective gear, placed barriers around the patient, started intravenous fluids, and transported him to the hospital. The employee's blood pressure in the ambulance and on the way to the hospital was $90 / 40 \mathrm{~mm} \mathrm{Hg}$. At the emergency department, the patient was assessed by a triage nurse in the ambulance and sent through a decontamination shower. After decontamination, the patient was given intravenous fluids. Drug and alcohol blood tests were normal. The patient was hospitalized and his hypotension improved with intravenous fluids. He was still slightly hypotensive upon discharge.

Upon presentation at the consultant's office, the patient was still hypotensive and vertiginous. He denied using any alcohol or products containing alcohol for the 2 years he had been applying hydrogen cyanamide. Research confirmed that the chemical was a growth regulator and that concomitant use of it and alcohol would produce profound hypotension. A report was made to the health department, using a state form, and appropriate workers' compensation documents filed. Private investigators and government investigations failed to reveal any alcohol use by the patient. A review of the patient's complete medical records failed to reveal any other cause of the symptomatology and physical findings. Motion pictures of the patient taken by a private investigator failed to reveal any alcohol or drug use. The consultant arranged for a "hold" tube of blood from the emergency department to be analyzed. The only abnormal substance discovered was hydrogen cyanamide at levels just above the detection limit. The patient was followed by the 
consultant for 6 weeks until his blood pressure returned to the normal range and his vertigo resolved. Three months after the incident the patient was working without symptoms. A repeated blood level of the chemical had the same results as the first one, demonstrating that trace amounts in the patient's blood was not the cause of the acute symptoms and physical findings. The worker was discharged as cured.

\section{Case Discussion}

The cause of the hypotension and vertigo was never fully delineated, even with a thorough workup, private investigator's investigation, and other tests. The patient was removed from exposure, decontaminated in the field and at the emergency department, and appropriately treated. A "hold" tube of blood showed only trace amounts of the substance, an anticipated finding. No specific treatment or antidote was necessary, other than intravenous fluids. By following proper procedures, the patient was treated without contaminating coworkers or emergency personnel.

\section{Diagnosis}

\section{History}

The history may be the only positive finding. It is important to list the precise symptoms and detail where and when they started. A precise record of when the symptoms started can establish a cause-and-effect relationship if they first occurred directly after a suspected exposure. Record what the patient was doing when the symptoms started and when he or she thought exposure occurred. When the patient thinks exposure occurred and what actually occurred may be more subjective than objectively true (Table 13.2).

Record the parameters of exposure. Was the patient working in a packinghouse and, if so, was the patient packing boxes of fruit or doing another job such as sorting out rotten fruit where the possibility of exposure to chemicals is greater? Was the patient operating equipment within a closed cab, becoming ill with no obvious exposure other than a strange odor? If the person was spraying in a closed cab and filtered air environment, there may be another cause of the illness such as influenza, food poisoning, or carbon monoxide poisoning from a leak from the exhaust pipes to the cab (Table 13.3).

If the person claims to have been exposed to a chemical, it is important to ask if the chemical was a liquid, solid, or gas, and what it smelled like. The color of the chemical is also important, because many chemicals are colorless but may have a distinctive odor. Ask if the patient actually saw the chemical or just assumed it was there. Sometimes the patient may be ill from another cause such as uncontrolled diabetes but may ascribe the illness to a chemical exposure. 
TABLE 13.2. Diagnosis and treatment.

\begin{tabular}{lc}
\hline Removal from exposure: & Physical examination: \\
Evacuate patient & Describe findings and how they \\
Retreat to a protected area & evolve over time \\
(closed building) & Other sources: \\
Don protective gear & Prior medical records \\
Decontamination: & Private investigator's reports \\
Remove contaminated clothing & Governmental investigations \\
and dispose of properly (as chemical & Eyewitness accounts \\
waste) & Media accounts \\
Shower and shampoo the patient & Employer accounts \\
carefully and completely & Research (see Table 13.4) \\
Protect medical handlers from secondary & Work-site visit (Chapter 11) \\
contamination & Decision making: \\
Treat acute symptoms: & Treatment: may not be necessary after \\
Cardiopulmonary resuscitation & removal from exposure and \\
Antidotes & decontamination \\
Antiemetics & Causation: \\
Anticonvulsants & Establish a most likely cause-and-effect \\
Cardiorespiratory support & relationship \\
When (or if initially) stable: & Look for internal consistency of the \\
Learn the accurate name or names & patient's history and physical \\
of the substances: & examination \\
Material Safety Data Sheet & External consistency with the research \\
(MSDS) & and other sources \\
Containers and labels & Impairments: \\
Application records & Based on objective findings \\
Poison control centers & and compared to AMA guidelines of \\
History: & evaluation of impairment \\
Was the worker in the vicinity of the & Disabilities: \\
chemicals being used? & Inability to perform social functions \\
Was there contact and exposure? & such as work \\
Was there poisoning and illness? & Treatment plan for further medical care: \\
Are there impairments? & Design a treatment plan for long term \\
Is there disability? & care, if necessary \\
\hline &
\end{tabular}

Ask for what purpose the chemical was being used and what is the normal use; the two uses may not be the same. Finally, ask about the progression of symptoms, and what made them better or worse. Since removal from exposure, have the symptoms improved?

It is important to ask when the person last ate before the onset of symptoms and if he or she was drinking alcoholic beverages, taking over-thecounter or prescription medicines, or using perfume. Fragrances can cause acute allergic symptoms; alcohol use while using certain growth regulators such as hydrogen cyanamide can cause an Antabuse reaction; and meals eaten at the site of contaminated fields can result in the accidental ingestion of chemicals (10-12).

Symptoms may vary by the formulation used, concentration, length of exposure, and personal protective equipment. For example, a person spraying 
TABLE 13.3. Differential diagnosis in pesticide exposure.

\begin{tabular}{ll}
\hline Irritant contact dermatitis & Bronchitis \\
Dermatitis, solvents & Pityriasis rosea \\
Dermatitis, other chemical & Folliculitis \\
Allergic contact dermatitis & Urticaria \\
Dermatitis, detergent & Insect bite \\
Dermatitis, plants and insects & Epistaxis \\
Scabies & Thyroid disease \\
Chicken pox & Drug misuse (legal \\
Drug eruption & and illegal) \\
Influenza & Food poisoning \\
Gastroenteritis & Carbon monoxide poisoning \\
Sinusitis & Mass psychogenic hysteria \\
Acne vulgaris & Fraud \\
Herpes zoster & Intentional homicide \\
Tinea cruris & Intentional suicide \\
Diabetes mellitus & Accidental suicide
\end{tabular}

Source: Data from Lessenger et al. (8) and Lessenger and Reese (15).

organophosphates may only have one or two of the classic symptoms if the exposure is minimal. On the other hand, a person with headaches, nausea, vomiting, chills, and fever may have influenza and not a chemically related disease, even if the patient had been spraying all day without protective equipment. It is important, especially when determining causation, that the patient's history be internally consistent and consistent with his workplace and work task, and with what is known about the chemicals $(9,13)$.

The differential diagnosis should be considered in each situation, and appropriate testing performed to rule out conditions such as diabetes, thyroid disease, and drug use that may mimic the symptoms of chemical exposure in an agricultural worker (Table 13.3) (8).

\section{Physical Examination}

The physical examination may not be helpful in determining if an exposure occurred. Rashes need to be carefully described and secondary changes due to scratching, infection, or treatment documented. Halogenated hydrocarbons can produce chloracne that may be confused with acne vulgaris in adolescents. Anhydrous ammonia can cause a characteristic hyperpigmented area after a burn heals. Petroleum products may cause irritative dermatitis. Scabies is common among farm workers. Allergic contact dermatitis may have a variety of causative agents (Table 13.3) $(1,13)$.

Inhalation of dusts, mists, and gases may cause instantaneous or delayed bronchospasms, producing a constellation of symptoms from wheezing to respiratory collapse. Nausea, vomiting, diarrhea, and abdominal pain can be the result of chemical exposure or from alternate illnesses such as diabetes, food poisoning, or influenza. Food poisoning is common in people who work 
in the fields and do not have the facilities to refrigerate their lunches. Anaphylaxis can result from envenomation by biting or stinging insects $(9,14)$.

Neurological symptoms such as burning, numbness, tingling, twitching, or seizures may be immediate or can be a delayed result of exposures. For example, low-level chronic exposure to organophosphates can cause a delayed polyneuropathy or memory loss (15).

Ocular symptoms are common in persons exposed to powders, dusts, sprays, or mists. After decontamination, the sclera may be erythemic. The corneas must be carefully examined for clouding or opacities.

\section{Laboratory Studies}

Urine, blood, or hair testing for specific offending chemicals is expensive and time-consuming, and must be collected immediately after the exposure to obtain reliable results. In many cases the patient may be cured or deceased before results are returned from reference laboratories. Nevertheless, when looking retrospectively at an alleged chemical illness to see if exposure actually occurred or if the illness can be attributed to a specific substance, a blood or urine test for the specific offending chemical can be helpful. Therefore, it is useful to draw an extra tube of blood in the laboratory or emergency department and freeze it for later testing $(1,8,9)$.

Blood, liver, and renal test results may be obscured by a preexisting disease and may be abnormal only in severe exposures. Nevertheless, such tests should be done as soon after the alleged exposure as possible to establish a baseline and to exclude other illnesses such as anemia, diabetes, thyroid disease, and infectious diseases. Rapid drug and alcohol tests can quickly document drug intoxication concomitant with, or masquerading as, a chemical related illness. Cholinesterase testing for organophosphate poisoning is discussed in Chapter 9 (16).

\section{Research}

Poison control centers are effective at calling employers, chemical companies, government agencies, or other entities to determine the exact name of the offending compound. These centers can also give a list of signs and symptoms to look for, assist in making a diagnosis, and advise on the latest treatment protocols (Table 13.4).

United States law mandates that employees exposed to chemicals must be given the Material Safety Data Sheet (MSDS) for any chemical with which they come in contact. Some employees may come into the emergency department or consultant's office with the MSDSs in hand along with container labels and other information. Container labels can give the precise chemicals and formulations to which the patient was exposed. Textbooks such as Ellenhorn's Medical Toxicology can be an invaluable aid. Online services through TOXLINE and PUBMED give timely and concise information on diagnosis 
TABLE 13.4. Information resources.

Ellenhorn MJ, Schonwald S, Ordog G, Wasserberger J. Ellenhorn's Medical Toxicology. Baltimore: Williams and Wilkins, 1997.

Reigart R, Roberts J. Recognition and Management of Pesticide Poisonings, 5th ed. Washington, DC: U.S. Environmental Protection Agency, 1999.

Tordoir W, Maroni M, He F. Health Surveillance of Pesticide Workers. Shannon: Elsevier, 1994.

Poison control centers

U.S. National Poison Control Hotline:

800-222-1222 (emergency)

202-362-3867 (administrative)

202-362-8561 (TDD)

National Library of Medicine.

PubMed: www.hcbi.nlm.nih.gov/PubMed/

United States Department of Health and Human Services, Public Health Service, Agency for Toxic Substances and Disease Registry. ATSDAR Tox Profiles, 2003.

National Pesticide Information Center:

800-858-7378

nptn@ace.orst.edu

Global Information Network on Chemicals (GINC), maintained by the National Institute of

Health Sciences, Japan. www.nihs.go.jp/GINC/

Material Safety Data Sheets (obtain from employer)

and treatment. The Agency for Toxic Substances and Disease Registry produces the ATSDR TOX Profiles, another helpful guide (Table 13.4) $(5,17,18)$.

\section{Site Visits}

Visits to the location where the exposure or event occurred can greatly assist the physician in learning what happened, where the patient or patients were, and how the exposure occurred. Sometimes it can be demonstrated that no exposure could have occurred and there was mass psychogenic hysteria or intentional fraud (see Chapter 11) (19).

\section{Case Study 2}

A 12-year-old boy was riding his bicycle to a fast-food restaurant when he slipped and fell in a shallow puddle of a foul-smelling liquid in the restaurant's parking lot. He became nauseous, vomited, and left the parking lot feeling dizzy and with blurred vision. He stumbled home, pushing his bicycle. A bystander smelled the puddle of liquid and notified the police who discovered that a farm vehicle had spilled a 50-gallon barrel of dicofol, a chlorinated hydrocarbon and DDT analogue. The barrel was spilled as a truck drove into the fast-food restaurant and the employees didn't bother to clean it up.

At home, the boy lay on his bed with his soaked clothing and slept. When his mother came home, she found him somnolent and insisted he shower. 
After the shower he felt better, but his mother laundered the clothing he was wearing, along with the bedding, and returned them to the boy's room. He continued to be symptomatic, even though she laundered the clothing several times. A week later the boy was referred to the consultant's office by the health department.

In the office, the boy was wearing clothing (not those he was wearing the day of the incident) that reeked of a hydrocarbon chemical. He was led to a back room and asked to disrobe. The clothing was taken outside and bagged. While the vital signs were normal, the boy was ataxic, demonstrated nystagmus, and his speech was slurred. His breath had a hydrocarbon odor. Samples of his blood, subcutaneous fat and urine were collected and were later found by a reference laboratory to be positive for dicofol. A complete blood count, urinalysis, and blood chemistries were all normal.

The boy's mother was asked to destroy all clothing, furniture, and appliances that had come in contact with the contaminated clothes the boy was wearing the day of the incident. This resulted in the destruction of the family's entire wardrobe, the boy's bed, a couch, and the washer. A report was made to the county health department as required by state law. A week after the decontamination, the ataxia, nystagmus, and hydrocarbon breath were gone. The slurred speech resolved in 2 weeks. A month after the exposure, a psychological test demonstrated cognitive and emotional deficiencies. A second test performed 2 months later was normal. Three months after decontamination, the boy was completely normal and he remained so for another 2 years of monitoring. No medication or treatment was given other than removal from exposure and decontamination.

\section{Case Discussion}

This case demonstrates the critical importance of removal from exposure and decontamination. Both were delayed in this case and the delay caused the persistence of the symptoms and the possible exposure of other family members. Once the patient was removed from exposure and the house properly decontaminated, his symptoms subsided. The boy was monitored for 2 years before the case was completely closed, but he suffered no residual impairments or disabilities once he was over the acute phase.

\section{Management}

\section{Presentation}

It is helpful to stage chemical injuries as mild, moderate, and severe based on symptoms and signs. Mild poisonings demonstrate few symptoms and normal vital signs. Moderate poisonings demonstrate more severe symptoms, objective signs, and normal vital signs. Severe poisonings demonstrate multiple complaints, objective signs, and unstable (or abnormal) vital signs. 
Mild and moderate poisonings can usually be evaluated on an outpatient basis. Once patients have been removed from exposure and decontaminated, they rarely require treatment other than an antiemetic for nausea and vomiting or topical steroids for a rash $(1,8)$.

Severe poisonings usually require hospitalization and intensive physiological support. Decontamination may include gastrointestinal lavage for accidental or deliberate ingestion. In cases of suicide or homicide, one or more poisons may be involved $(2,12)$.

\section{Removal from Exposure}

Whether the patient is seen in the emergency department or the office, it is imperative that the patient be removed from exposure until the symptoms and causes of the illness can be diagnosed and decontamination assured. Removal from exposure may not equate with complete removal from work. It may be possible to return the employee to modified duty while the workup and treatment are in progress. In mass causality situations, evacuation to a safe location or sequestration in a secure building with the windows closed and the air conditioning off may be necessary to prevent further inhalation of the agents $(4,12)$.

\section{Resuscitation}

In emergent cases where the vital signs are unstable and there is respiratory arrest or cardiac arrest, aggressive resuscitation using the standard protocols is indicated. If the patient is unstable with abnormal vital signs, decontamination can be carried out simultaneously with resuscitation $(1,3,4)$.

\section{Decontamination}

Decontamination includes removal of the offending chemical from the person, clothing, and personal protective equipment. Decontamination of the person should include a thorough irrigation of the eyes if they have been subjected to any exposure. The sooner the eyes are irrigated, the less damage that will occur and workers are taught to use eye irrigation stations near their work site in the event of eye exposure. Careful attention should be paid to scalp hair, the axillae, and pubic hair because they are usually ignored in decontamination. Improved decontamination can be carried out if screens or other mechanisms are used to ensure privacy when exposed workers disrobe. Medical personnel are at risk of exposure during decontamination and should use protective measures, including chemical-resistant gloves $(2,12)$.

If the offending chemical is consumed, typically by eating contaminated food in the fields, it may be necessary to decontaminate the gut. Recalling that many agricultural chemicals are based on hydrocarbons, activated char- 
coal may be the best method so that vomiting won't be induced, with its accompanied risk of aspiration into the lungs (12).

It is typically impossible to decontaminate contaminated clothing. If they are laundered, the laundry machines can become contaminated. They are typically placed in plastic bags as chemical waste to be disposed of in compliance with local rules, either by incineration or removal to a hazardous waste depository.

\section{Antidotes}

Antidotes such as atropine, pralidoxime, and vitamin $\mathrm{K}$ are rare. In China, studies have demonstrated that the use of antidotes brought people near death when they didn't have a pesticide injury. Atropine and pralidoxime are useful as antidotes in organophosphate and carbamate poisoning when they are used to treat bradycardia and hypotension, and in drying up copious secretions (Table 13.5) $(4,17,20)$.

TABLE 13.5. Antidotes and specific treatments.

\begin{tabular}{|c|c|c|}
\hline Atropine & $\begin{array}{l}\text { Organophosphate } \\
\quad \text { or carbamate poisoning }\end{array}$ & $\begin{array}{l}\text { Adults: } 2-4 \mathrm{mg} \text { every } 10-15 \text { minutes } \\
\text { to symptoms, i.v. } \\
\text { Children: } 0.015 \text { to } 0.5 \mathrm{mg} / \mathrm{kg} \text { every } \\
15 \text { minutes to symptoms, i.v. }\end{array}$ \\
\hline $\begin{array}{l}\text { Pralidoxime } \\
\text { (2PAM) } \\
\text { Protopam }^{\mathrm{TM}}\end{array}$ & $\begin{array}{l}\text { Organophosphate and } \\
\text { carbamate poisoning } \\
\text { (cholinesterase } \\
\text { reactivant) }\end{array}$ & $\begin{array}{l}\text { Adults: } 4 \mathrm{mg} / \mathrm{kg} \text { over } 4 \text { to } 6 \text { hours or } \\
8 \text { to } 10 \mathrm{mg} / \mathrm{kg} / \mathrm{hr} \text {, i.v. } \\
\text { or } 1 \text { to } 2 \mathrm{~g} \text { over } 15 \text { to } 30 \text { minutes, i.v., } \\
\text { repeated every } 1 \text { to } 2 \text { hours by } \\
\text { symptoms } \\
\text { Children: } 25 \mathrm{mg} / \mathrm{kg} \text { loading dose, } \\
\text { followed by } 20 \mathrm{mg} / \mathrm{kg} / \mathrm{hr} \text { until } \\
\text { symptoms have abated }\end{array}$ \\
\hline $\begin{array}{l}\text { Vitamin } \mathrm{K} \\
\text { Phytonadione }^{\mathrm{TM}} \\
\text { Mephyton }^{\mathrm{TM}} \\
\text { AquaMephyton }^{\mathrm{TM}}\end{array}$ & Coumadin (Rodenticide) & $\begin{array}{l}\text { Adults: } 10 \text { to } 25 \mathrm{mg} / \mathrm{kg} \text { p.o. or } 2 \\
\text { to } 5 \mathrm{mg} \text { p.o. } \\
2.5 \text { to } 25 \mathrm{mg} \text { s.q. or i.m. (or rarely up } \\
\text { to } 50 \mathrm{mg} \text { ) every } 6 \text { hours to } \\
\text { symptoms } \\
\text { Children: } 5 \text { to } 10 \mathrm{mg} / \mathrm{kg} \text { p.o. or } 2.5 \text { to } \\
25 \mathrm{mg} \text { s.q. or i.v. every } 6 \text { hours adjusted } \\
\text { to severity of symptoms }\end{array}$ \\
\hline Physostigmine & $\begin{array}{l}\text { Muscarinic effects of } \\
\text { anticholinesterase } \\
\text { chemicals; use in } \\
\text { situations of severe } \\
\text { poisoning and excessive } \\
\text { agitation, long-lasting } \\
\text { seizures, and cardiac } \\
\text { arrhythmias }\end{array}$ & $\begin{array}{l}\text { Adults: } 2 \mathrm{mg} \text { i.v. and } 1 \text { to } 2 \mathrm{mg} \text { every } \\
20 \text { minutes until response } \\
\text { Children: } 0.02 \mathrm{mg} / \mathrm{kg} \text { i.v. }\end{array}$ \\
\hline
\end{tabular}


TABLE 13.5. Antidotes and specific treatments. (continued)

\begin{tabular}{ccc}
\hline Diazepam & Seizures in cholinesterase & Adults: 5 to $10 \mathrm{mg}$ i.v., s.q., or rectally \\
& and carbamate poisoning & Children: 1 to $5 \mathrm{mg}$, i.v., s.q., or rectally \\
Perchlorperazine & Antiemetic & Adults: 5 to $10 \mathrm{mg}$, p.o. every 6 to \\
Compazine & & 8 hours, 5 to $10 \mathrm{mg}$ i.m. every 6 \\
& & to 8 hours, \\
& & 2.5 to $10 \mathrm{mg}$ i.v. every 6 to 8 hours \\
& to symptoms \\
& Children: Not indicated under 2 years. \\
& Over 2 years of age: $0.06 \mathrm{mg} / \mathrm{lb}$ every \\
& 6 to 8 hours \\
Promethazine & Antiemetic & Adults: $25 \mathrm{mg}$ p.o. or i.m. every \\
(Phenergan) & & 4 to 6 hours \\
& & Children: $0.5 \mathrm{mg} / \mathrm{lb}$ every 4 to 6 hours \\
& i.m. or p.o. \\
\hline
\end{tabular}

Source: Data from Tordoir et al. (4) and Ellenhorn et al. (17).

\section{Reports}

In jurisdictions where required, reports must be made to the workers' compensation insurance carrier and appropriate government agency.

\section{Follow-Up}

Serial examinations to follow chronic sequelae of chemical exposures may be necessary. Work impairments and disability status require documentation on an ongoing basis, especially if the patient is receiving disability payments. A long-term treatment plan may be required by insurance companies.

\section{References}

1. Lessenger JE. The pesticide-exposed worker: an approach to the office evaluation. J Am Board Fam Pract 1993;6:33-41.

2. O'Malley M. Clinical evaluation of pesticide exposure and poisonings. Lancet 1997;349:1161-6.

3. Jones TF, Craig AS, Hoy D, et al. Mass psychogenic illness attributed to toxic exposure at a high school. N Engl J Med 2000;342:96-100.

4. Tordoir W, Maroni M, He F. Health Surveillance of Pesticide Workers. Shannon: Elsevier, 1994.

5. Reigart R, Roberts J. Recognition and Management of Pesticide Poisonings, 5th ed. Washington, DC: U.S. Environmental Protection Agency, 1999.

6. Schiffman SS, Walker JM, Dalton P, et al. Potential health effects of odor from animal operations, wastewater treatment and recycling of byproducts. J Agromed 2000;7:7-81.

7. Simpson WM, Brock CD. Chemophobia, family medicine, and the doctor-patient relationship. J Agromed 2003;9:7-16. 
8. Lessenger JE, Estock MD, Younglove T. Analysis of 190 cases of suspected pesticide illness. J Am Board Fam Pract 1995;8:278-82.

9. Agency for Toxic Substances and Disease Registry. Obtaining an exposure history. Am Fam Physician 1993;48:483-91.

10. Lessenger JE. Occupational acute anaphylactic reaction to assault by perfume spray in the face. J Am Board Fam Pract 2001;14(2):137-40.

11. Lessenger JE. Case study: hypotension, nausea and vertigo linked to hydrogen cyanamide exposure. J Agromed 1998;5(3):5-11.

12. Kales SN, Christiani DC. Acute chemical emergencies. N Engl J Med 2004;350:800-8.

13. Danse IR. Common Sense Toxics in the Workplace. New York: Van Nostrand Reinhold, 1991.

14. Waller K, Prendergast TJ, Slagle A, Jackson RJ. Seizures after eating a snack food contaminated with the pesticide Endrin. West J Med 1992;157:648-51.

15. Lessenger JL, Reese BE. The pathophysiology of acethlcholinesterase inhibiting pesticides. J Agromed 2000;7:5-19.

16. Lessenger JE, Reese BE. The rational use of cholinesterase testing in pesticide poisoning. J Am Board Fam Pract 1999;12:307-14.

17. Ellenhorn MJ, Schonwald S, Ordog G, Wasserberger J. Ellenhorn's Medical Toxicology, 2nd ed. Baltimore: Williams and Wilkins, 1997.

18. Agency for Toxic Substances and Disease Registry. ASTAR Tox Profiles. Atlanta: Department of Health and Human Services, 2003.

19. Lessenger, JE. Case report: fraudulent pesticide injury: value of the work site visit. J Agromed 1996;3:27-32.

20. He F, Wang S, Liu L, Chen S, Zhang Z, Sun J. Clinical manifestations and diagnosis of acute pyrethroid poisoning. Arch Toxicol 1989;63:54-8. 